\title{
Bioelectrical impedance analysis for monitoring cancer patients receiving chemotherapy and home parenteral nutrition
}

\author{
Paolo Cotogni $i^{1,2^{*}}$, Taira Monge ${ }^{2,3}$, Maurizio Fadda ${ }^{3}$ and Antonella De Francesco ${ }^{3}$
}

\begin{abstract}
Background: Home parenteral nutrition (HPN) can improve survival, quality of life, nutritional and functional status in cancer patients. Bioelectrical impedance analysis (BIA) is a non-invasive, validated method to assess body composition. The objective of this prospective single-arm study was to investigate the impact of HPN in advanced cancer patients receiving chemotherapy assessed by BIA, clinical and laboratory measures.

Methods: Adult malnourished cancer outpatients with solid tumors receiving anticancer treatments who were candidates for daily HPN were enrolled. Patients were assessed at baseline (T0), 60 (T1) and 90 days (T2) after HPN start. Assessments included anthropometric and clinical-oncological characteristics, performance status, inflammatory response and Patient-Generated Subjective Global Assessment (PG-SGA).

Results: Sixty-five advanced cancer patients were enrolled. Median overall survival was 317 days. Body weight, $\mathrm{BMI}$, oral calorie and protein intake increased over time $(P<0.01)$. At T2 the proportion of well-nourished patients, Karnofsky performance status and modified Glasgow prognostic score were improved $(P<0.01)$, total body water was reduced $(P=0.04)$, and fat mass increased $(P=0.04)$. Reactance, resistance and phase angle were significantly associated with survival at T0, T1, and T2, respectively. At T2, PG-SGA category A was a predictor of survival $(P<0.0001)$.

Conclusions: After 90 days of HPN, patients experienced significantly improved nutritional status, performance status, prognostic score and some BIA measures. HPN may be an important therapy in oncology patients receiving chemotherapy. Longitudinal use of BIA may help track the effects of HPN and disease progression, potentially contributing to optimal global patient management.
\end{abstract}

Keywords: Cancer, Bioelectrical impedance analysis, Body composition, Malnutrition, Home parenteral nutrition

\section{Background}

Malnutrition is commonly reported in cancer patients and can increase mortality, morbidity and treatment complications, and can reduce treatment effectiveness and quality of life (QoL) [1, 2]. Cancer patients are usually at risk of developing cachexia, which is associated

\footnotetext{
* Correspondence: paolo.cotogni@unito.it

1 Department of Anaesthesia and Intensive Care, Pain Management and

Palliative Care, S. Giovanni Battista Hospital, University of Turin, C.so Bramante 88, 10126 Turin, Italy

${ }^{2}$ Unit of Parenteral Nutrition in Oncology, S. Giovanni Battista Hospital, C.so Bramante 88, 10126 Turin, Italy

Full list of author information is available at the end of the article
}

with progressive weight loss, loss of muscle and fat tissue, impaired immune function, and metabolic disturbances caused by a variable combination of reduced food intake, systemic inflammation and abnormal metabolism [3, 4]. Chemotherapy can cause side effects that further compromise nutritional status including nausea, vomiting, anorexia, and loss of body weight. Many studies clearly demonstrated that muscle loss strongly predicts the development of chemotherapy toxicity $[5,6]$. Early nutritional intervention is critical to optimal patient outcomes [7], yet most patients do not receive

(c) The Author(s). 2018 Open Access This article is distributed under the terms of the Creative Commons Attribution 4.0 International License (http://creativecommons.org/licenses/by/4.0/), which permits unrestricted use, distribution, and reproduction in any medium, provided you give appropriate credit to the original author(s) and the source, provide a link to the Creative Commons license, and indicate if changes were made. The Creative Commons Public Domain Dedication waiver (http://creativecommons.org/publicdomain/zero/1.0/) applies to the data made available in this article, unless otherwise stated. 
intense nutritional therapy until they experience significant weight loss [8-10].

Parenteral nutrition (PN) has been shown to improve survival, QoL, and nutritional and functional status, and slow progressive weight loss in advanced cancer patients [11-18]. Current guidelines recommend home parenteral nutrition (HPN) for cancer patients with chronic defects of dietary intake or absorption when enteral nutrition is not sufficient or feasible, if they are expected to die from starvation prior to cancer spread [19-21].

Clinicians must identify patients likely to benefit from $\mathrm{PN}$, and balance the potential to improve nutritional status, QoL and survival with PN without prolonging life in patients with no chance of improvement [20,22]. Some key clinical measures may not accurately reflect patient status due to inflammation and disease state. Therefore, clinicians must rely on multiple characteristics such as Karnofsky performance status (KPS), tumor site and spread, and Glasgow prognostic score (GPS) in addition to anthropometric measures to assess patient status and prognosis to guide nutrition management $[23,24]$.

Cancer patients experience not only loss of overall body weight but also loss of muscle tissue and body cell mass (BCM) [25], and changes in fluid distribution with extracellular expansion and reduced intracellular water. Managing nutritional needs based only on body weight can be misleading, as it does not reflect body composition [26, 27].

Bioelectrical impedance analysis (BIA) is non-invasive, validated method to assess body composition and nutritional status, which offers an efficacious way to track changes in body composition over time [28]. Bioelectrical impedance vector analysis (BIVA) is a pattern analysis of impedance measurements (resistance and reactance) plotted as a vector in a coordinate system [29] that can be used as a quality control measure for interpretation of BIA results. It is useful in assessing hydration status, which is difficult to determine based on biochemical methods and physical exam [30].

BIA assessments such as phase angle (PA) and fat free mass (FFM) have been correlated with nutritional status and survival in cancer patients [26, 31-34]. However, few published studies have examined the utility of body composition obtained from BIA to monitor the effects of nutritional support in cancer patients on HPN $[15,17]$.

The primary objective of the current study was to investigate the impact of HPN in advanced cancer patients receiving chemotherapy using BIA measures along with clinical and laboratory measures by a prospective study without a control group. The secondary objective was to evaluate the association between BIA measures and other clinical and laboratory variables with survival.

\section{Methods}

\section{Study design}

This prospective, longitudinal study was conducted in a 1200-bed tertiary university hospital. From October 1, 2014 through March 31, 2015, all adult cancer outpatients with solid tumors receiving anticancer treatments (neoadjuvant/adjuvant or palliative chemotherapy with or without radiation therapy) who were candidates for daily HPN based on European guidelines were eligible for enrollment. Criteria for HPN included: proven and prolonged failure to meet nutrition requirements by oral or enteral route, with impending risk of death due to malnutrition; life expectancy $>2$ months; KPS $>50$; control of pain; absence of severe organ dysfunctions; written informed consent confirming that the patient accepted this modality of nutrition support; approval by the physician responsible for HPN, the oncologist and the general practitioner; presence of environmental conditions compatible with HPN; availability of an in-home caregiver; and availability of a specifically trained nursing team dedicated to the patient home care, as provided by the Public Health Service [35]. Ethics Committee approval was obtained prior to study start, and written informed consent was obtained from each patient prior to any study procedures.

Patients were assessed in a hospital setting by the physician responsible for HPN and the dietician at baseline (T0), at 60 days (T1), and 90 days (T2) after the start of HPN. Data recorded included anthropometric (actual body weight, weight loss, body mass index [BMI]) and clinical-oncological (tumor site and stage, anticancer treatments) assessments. Performance status was graded using the WHO/Eastern Cooperative Oncology Group $(E C O G)$ scale $(0=$ normal performance, $4=$ bed-bound) [36] and KPS (0-100) [37]. Systemic inflammation was estimated using serum $\mathrm{C}$-reactive protein (CRP) and albumin, and inflammatory response was graded according to the modified Glasgow Prognostic Score (mGPS) (0-2) that is highly predictive of morbidity and mortality in cancer patients [38]. At baseline (T0), oral intake was assessed quantitatively using a 24-h food recall by the dietician through a structured interview in which patients were asked to recall all food and drink they consumed in the previous $24 \mathrm{~h}$. Nutrition was assessed using the Patient-Generated Subjective Global Assessment (PG-SGA), which combines qualitative and semi-quantitative data to yield a comprehensive 'malnutrition score' (A = well-nourished; $\mathrm{B}=$ suspected malnutrition or moderate malnutrition; or $\mathrm{C}=$ severely malnourished) [39]. The PG-SGA was developed specifically for patients with cancer and it includes questions regarding the presence of nutritional symptoms and short-term weight loss. It was designed so that the components of the medical history can be completed by the 
patient using a check box format. In particular, the questions regard current weight, weight history, and acute weight changes; any changes in food intake over the past month; any nutrition impact symptoms experienced over the previous 2 weeks; and any changes to activities and functions over the previous month. Conversely, the physical examination is performed by a physician or dietitian. Start date, end date, and management of HPN were recorded over time.

After the start of HPN, all patients were closely monitored by the physician responsible for HPN through regularly scheduled and structured telephone interviews (at least every 15 days) and home visits by the nursing team and general practitioner (initially daily for 23 weeks, and at least every 7 days thereafter). After adequate training, home caregivers administered HPN. HPN was delivered overnight for 10-14 h per day, using standard commercially manufactured ready to use bags containing amino acids, electrolytes, glucose, and lipids. HPN was prescribed to provide $20-25 \mathrm{kcal} / \mathrm{kg} /$ day for bedridden or $25-30 \mathrm{kcal} / \mathrm{kg} /$ day for ambulatory patients and $1-1.5 \mathrm{~g} / \mathrm{kg} /$ day amino acid. Every 30 days from HPN start ( \pm 5 days), an outpatient re-evaluation by both the physician and the dietitian (including a 24-h food recall) was performed. Overall survival was calculated as the number of days between the date of HPN start and the date of patient death from any cause, with censoring at the date of last follow-up assessment in alive subjects (at November 30, 2016).

\section{Bioelectrical impedance analysis}

Tetrapolar single frequency BIA is performed by passing current between two surface electrodes placed on the right hand and right foot [40]. The primary carriers of current in tissue are charged ions, such as sodium or potassium therefore conductivity of blood and urine is high, muscle is intermediate, and bone, fat, and air is low. Cell membranes form barriers to the flow of charge, therefore intact cell membranes cause increased resistance to flow [41]. BIA actually measures the voltage (V) produced between two electrodes located near to, but different from, the sites where current is introduced in most cases. The measurement normally is called impedance (Z), which is made up of resistance (RZ) and reactance (XC). Resistance reflects opposition to current due to body fluids and is therefore a measure of cellular hydration, and reactance reflects opposition due to cell membranes and tissues, and indicates cellular integrity. PA is calculated as the arctangent of the ratio of reactance divided by resistance. PA is considered a measurement of overall health [30] and decreases over time due to age, disease, and sedentary lifestyle. BIA does not directly measure total body water (TBW), but rather a weighted sum of extracellular water $(\mathrm{ECW})$ and intracellular water (ICW) resistivity, which allows FFM and TBW to be estimated.

All BIAs were performed by the same dietitian using an impedance plethysmograph that emits an 800-A, $50-\mathrm{kHz}$ alternating current (STA-BIA $50-\mathrm{kHz}$, Akern, Florence, Italy). BIAs were performed with an empty bladder, after fasting and not receiving HPN for at least $8 \mathrm{~h}$, with patient lying supine on a bed with legs apart and arms not touching the torso. The four standard electrodes were positioned on the ulnar aspect of the right wrist and the right medial malleolus, according to the standardized technique [41].

BIA was performed at T0, T1, and T2. The following parameters of BIA were evaluated: RZ, XC, PA, ECW, ICW, TBW, FFM, fat mass (FM), BCM, extracellular mass (ECM), ECM/BCM ratio, and muscle mass. BIVA was plotted at each time point.

\section{Statistical analysis}

Statistical analysis calculated changes in patient and BIA measures over time. Results for continuous variables were expressed as mean, median and range, and categorical variable as counts and percentage. For variables assessed at T0, T1, and T2, the values for a single variable were plotted per patient, a line was fitted, and the slope was calculated. A t-test was performed to determine whether the mean slope was significantly different from 0 for each variable. Variables assessed at T0 and T2 were analyzed with paired Student's t-test, and categorical variables were analyzed with Wilcoxon test for paired sample and Mann-Whitney test for independent samples. Survival data were summarized by computing the overall survival curve with the Kaplan-Meier method. The comparison of survival between curves was assessed by log-rank and Wilcoxon test. Hazard ratio (HR) with 95\% confidence interval (CI) was also calculated for survival data. Assessment of patient and BIA variables associated with survival was performed using Cox proportional-hazards regression. The accuracy of a test to discriminate diseased cases from normal cases was evaluated using Receiver Operating Characteristic (ROC) curve analysis. A two-sided $P$-value of 0.05 or less was considered significant. All analyses were performed using SPSS Statistics Release 23.0.0 (SPSS, Inc., an IBM Company, Chicago, IL).

\section{Results}

\section{Patients}

Sixty-five malnourished stage III and IV adult cancer patients were consecutively enrolled in the study. Thirty-two (49\%) were female, and median age was 64 years (range 33-79). Tumor locations across the population were stomach: $26(40 \%)$, pancreas/biliary system: 15 (23\%), colon/rectum: 9 (14\%), gynecological: 5 
(8\%), esophagus: 3 (4\%), and other: 7 (11\%). Forty-six patients $(71 \%)$ had metastasis, and $16(25 \%)$ were diagnosed with stage III cancer, and 49 (75\%) were diagnosed with stage IV cancer. All patients were receiving chemotherapy (neoadjuvant: 9, adjuvant: 37 , and palliative; 19, respectively) and 10 received both chemotherapy and radiation therapy. Adjuvant chemotherapy was given in 37 patients following surgery, while neoadjuvant chemotherapy in 5 patients that subsequently underwent surgery. Overall, 42 patients $(65 \%)$ received surgery. Median percent weight loss in the 3 months prior to the start of HPN was 10.7\% (range 1.9-27.8\%). At baseline (T0), all patients had residual but insufficient oral food intake (a median of $600 \mathrm{Kcal}$ ), and supplemental HPN provided a median of 1200 and $1000 \mathrm{kcal}$ per day at T1 and T2 respectively.

No HPN-related metabolic complications occurred. The incidence of catheter-related bloodstream infections (CRBSI) was 0.27 per 1000 catheter-days. The overall rate of catheter-related complications (i.e., mechanical, CRBSI, and venous thrombosis) was 1.06 per 1000 catheter-days. No patients required hospitalization due to complications.

\section{Clinical and BIA measures}

Table 1 shows patient and BIA variables at T0, T1, T2, and the calculated mean slopes for variables with assessments at all three time points. Actual body weight and BMI, as well as oral calorie and protein intake had significantly positive slopes, and the proportion of patients with BMI $\leq 18.5 \%$ decreased from 33.8 to $30.7 \%$ from baseline to T2. At T2 a greater proportion of patients qualified as PG-SGA category A (well-nourished), and KPS and mGPS were significantly improved. The proportion of patients with KPS $\geq 70$ increased from 66.2 to $77 \%$ at from baseline to T2. Among BIA variables, TBW had a significantly negative slope, and FM had a significantly positive slope from baseline to T2.

Table 2 shows patient and BIA variables subsetted by PG-SGA at T0 (B or C; entry criteria prohibited category A). In both cohorts, actual body weight, BMI, oral calorie and protein intake had significantly positive slopes, but the positive slopes trended towards being steeper in the group with PG-SGA of B at study entry. There were multiple variables with significant changes in the group with PG-SGA of B at study entry, but not PG-SGA of C at entry: increase in KPS and albumin, decrease in ECOG, decrease in TBW, and increase in FM.

BIVA summary plot for $\mathrm{T} 0, \mathrm{~T} 1$, and $\mathrm{T} 2$ is shown in Fig. 1, and detailed distribution at each timepoint are shown in Fig. 1b, c and d. At T0, patients presented with altered body composition with reduced muscle mass and increased total body water. At T1, body composition improved with reduction in water and increase in soft tissue, and at T2 body composition declined. Distribution across all quadrants is seen in Fig. 1b, c and d, signifying a wide variety in patient status and disease progression.

\section{Survival}

All patients were alive at T2, and at the time of analysis, 11 of the 65 patients enrolled were still alive. No patients were lost to follow-up. Figure 2 details overall survival probability, with median overall survival 317 days [range 92-790; 95\% CI 219-415]. No HPN-related mortality occurred. Overall, 28 patients (43.1\%) survived one-year or greater, and five patients $(7.7 \%)$ survived 2 years or greater. However considering the 6-month enrollment window and the data analysis cut-off date, only 23 of the 65 patients enrolled could have potentially reached 2 -year survival therefore the 2-year survival rate in eligible patients was $21.7 \%$.

Table 3 shows BIA variables associated with survival according to Cox regression analysis. $\mathrm{XC}, \mathrm{RZ}$, and $\mathrm{PA}$ were significantly associated with survival at $\mathrm{T} 0, \mathrm{~T} 1$, and T2, respectively. Among the patient variables, only PG-SGA category A at T2 (SE 1.510, P 0.014, 95\% CI 0.001-0.465) was associated with survival. This result was confirmed by ROC curve (SE 0.076, $P$ 0.006, 95\% CI $0.614-0.911)$ also in terms of sensibility $(80 \%)$, specificity (59\%), and accuracy (76\%).

At T2, the comparison of survival between curves assessed by log-rank test showed that PG-SGA category A (median duration days: 652; CI 635-660) was a predictor of survival $(P<0.0001)$; conversely, the PG-SGA category $C$ (median duration days: 162; CI 102-222) was a predictor of mortality vs. both $\mathrm{A}$ and $\mathrm{B}$ categories $(P=$ 0.003 and $P<0.0001$, respectively) (Fig. 3). Assessing PG-SGA category at T2 as a predictor of survival, the HR for category A vs. B was 0.39 (CI 0.17-0.90); for category A vs. C was 0.15 (CI 0.06-0.37), and for category $\mathrm{B}$ vs. $\mathrm{C}$ was 0.41 (CI $0.20-0.72$ ). The proportion of patients with improvements, declines, or no change in PG-SGA from T0 to T2 and the corresponding survival time is shown in Table 4. In rank order, patients who improved from category B to A had the longest median survival time, and patients who declined from category $\mathrm{B}$ to $\mathrm{C}$ had the shortest median survival time.

The comparison of survival between curves showed that no metastasis (median duration days: 487; CI 88$894)$ vs. any metastasis (median duration days: 213; CI 163-263) was also a predictor of survival $(P=0.002)$ (Fig. 3b). Assessing no metastasis as a predictor of survival, the HR was 0.37 (CI 0.21-0.63).

\section{Discussion}

Improving nutritional status is critical in cancer patients: treating malnutrition has been shown to improve 
Table 1 Changes in patient and BIA variables over time

\begin{tabular}{|c|c|c|c|c|c|}
\hline & TO & $\mathrm{T} 1$ & $\mathrm{~T} 2$ & $\begin{array}{l}\text { Mean Slope } \\
\text { (change per day) }\end{array}$ & $P$ \\
\hline \multicolumn{6}{|l|}{ Patient Variables } \\
\hline Actual body weight, kg & 57.4 [57.0] (33.0-100.0) & 58.9 [57.0] (37.7-96.0) & 59.7 [60.0] (37.5-95.0) & 0.02 & $<0.01$ \\
\hline Body Mass Index, $\mathrm{kg} / \mathrm{m}^{2}$ & $21.2[21.1](12.7-39.1)$ & 21.7 [21.4] (15.4-37.5) & 22.0 [21.7] (15.4-37.1) & 0.01 & $<0.01$ \\
\hline Oral calorie intake, kCal & 597 [600] (0-1500) & 904 [1000] (0-2550) & 959 [1000] (0-2550) & 4.18 & $<0.01$ \\
\hline Oral protein intake, g/day & $21.1[20.0](0-50)$ & 32.2 [35.0] (0-110) & $34.5[40.0](0-90)$ & 0.15 & $<0.01$ \\
\hline \multirow[t]{3}{*}{ PG-SGA category, \% } & A: $0 \%$ & NA & A:8\% & NA & $<0.01$ \\
\hline & B: $48 \%$ & & B:48\% & & \\
\hline & C: $52 \%$ & & C: $44 \%$ & & \\
\hline Karnofsky PS & 67.4 [70.0] (50-90) & NA & 72.5 [70.0] (50-90) & NA & $<0.01$ \\
\hline ECOG PS & $1.3[1](0-2)$ & NA & $1.2[1.0](0-2)$ & NA & 0.09 \\
\hline Albumin, g/dL & $3.4[3.5](1.9-4.5)$ & NA & $3.5[3.5](2.2-4.6)$ & NA & 0.19 \\
\hline C-reactive protein, mg/L & 16.7 [10.9] (0.4-73.2) & NA & 13.5 [6.9] (0.1-140) & NA & 0.23 \\
\hline \multirow[t]{4}{*}{ mGPS } & $1[1](0-2)$ & NA & $0.6[0](0-2)$ & & 0.01 \\
\hline & $0: 37 \%$ & & $0: 62 \%$ & & \\
\hline & $1: 26 \%$ & & 1: $14 \%$ & & \\
\hline & $2: 37 \%$ & & $2: 25 \%$ & & \\
\hline \multicolumn{6}{|l|}{ BIA Variables } \\
\hline Resistance, Ohm & 590.9 [606.0] (401-804) & 586.6 [586.0] (401-898) & 584.0 [607.0] (372-780) & -0.08 & 0.45 \\
\hline Reactance, Ohm & 52.0 [48.0] (19-120) & 51.0 [49.0] (20-99) & $51.2[46.0](22-116)$ & -0.01 & 0.68 \\
\hline Phase Angle, ${ }^{\circ}$ & $4.8[4.8](2.5-8.1)$ & 5.0 [4.7] (2.5-11.4) & 5.0 [4.5] (2.3-10.0) & 0.00 & 0.32 \\
\hline Total Body Water, \% & 59.4 [59.7] (39.3-85.8) & 58.6 [58.5] (41.3-76.6) & 58.0 [58.2] (41.7-76.4) & -0.02 & 0.04 \\
\hline Extracellular Water, \%, & 52.1 [52.2] (25.4-70.7) & 52.1 [52.6] (29.5-69.9) & 52.2 [53.5] (32.3-73.2) & 0.00 & 0.98 \\
\hline Intracellular water, \% & 47.9 [47.8] (29.3-74.6) & 48.1 [47.7] (30.1-70.5) & 47.7 [46.3] (26.8-67.7) & 0.00 & 0.89 \\
\hline Fat Mass, \% & $23.3[23.2](-7.1-50.8)$ & 24.1 [24.6] $(0.5-48.4)$ & 25.8 [24.3] (1.6-76.9) & 0.03 & 0.04 \\
\hline Fat Free Mass, \% & 74.6 [76.6] (49.2-86.9) & 76.0 [75.4] (51.6-101.2) & 75.0 [75.7] (52.5-98.4) & 0.01 & 0.52 \\
\hline Muscle Mass, \% & 44.0 [39.2] (25.3-75.4) & 43.2 [40.0] (19.5-120.7) & 42.1 [38.9] (23.5-81.3) & -0.02 & 0.37 \\
\hline
\end{tabular}

All values are expressed as mean [median] (range), or percentage as indicated

TO: at start of HPN; T1: 60 days after the start of HPN; T2: 90 days after the start of HPN

NA Not applicable, BIA Bioelectrical impedance analysis, BMI Body mass index, ECM Extracellular mass, BCM Body cell mass, PS Performance status, ECOG Eastern cooperative oncology group, mGPS Modified Glasgow prognostic score, PG-SGA Patient-generated subjective global assessment; Category A = well-nourished; Category $B=$ suspected malnutrition or moderate malnutrition; Category $C=$ severely malnourished

symptoms including fatigue and appetite loss [42, 43]; improve the effectiveness of chemotherapy by reducing the likelihood of toxicity [17]; improve patient QoL [4245]; and improve survival [16, 18].

In the current study, HPN not only provided a source of calories but also was associated with an increased oral intake with a $50 \%$ increase in oral calorie and protein intake after 90 days, possibly due to improved patient appetite and decreased side effects of the disease and treatments. This is consistent with previous studies investigating $\mathrm{HPN}$ in cancer patients, which reported a decrease in fatigue and appetite loss after 3 months of HPN [42, 43], as well as an association between HPN and improved nutritional status, QoL, and KPS [42].

Several studies evaluating patients undergoing chemotherapy found that not only was sarcopenia (i.e., loss of lean body mass) an independent risk factor for dose-limiting toxicities, hospitalization and survival, but patients who were malnourished at the start of treatment experienced a further nutritional decline during chemotherapy $[31,46]$. Considering $100 \%$ of the advanced cancer patients enrolled in the current study were undergoing chemotherapy during the study period, the improvement in nutritional status is especially noteworthy.

The study supports the utility of BIA in the management of cancer patients. BIA may be useful for an accurate evaluation of effects of HPN over time [47]. Use of weight alone to assess effects of $\mathrm{PN}$ can be of limited value due to several confounding factors (e.g., edema, ascites, fluid retention), but BIA can provide valuable data on changes in body compartments, which can help 
Table 2 Changes in patient and BIA variables over time by PG-SGA at TO

\begin{tabular}{|c|c|c|c|c|c|c|}
\hline & PG-SGA at TO & T0 & $\mathrm{T} 1$ & T2 & $\begin{array}{l}\text { Mean Slope } \\
\text { (change per day) }\end{array}$ & $P$ \\
\hline \multicolumn{7}{|l|}{ Patient Variables } \\
\hline \multirow[t]{2}{*}{ Actual body weight, kg } & B & $60.2[57.5](39.0-100.0)$ & 62.3 [59.6] (41.5-96.0) & 62.8 [60.0] (43.5-95.0) & 0.03 & 0.01 \\
\hline & C & $54.9[56.5](33.0-85.5)$ & 55.7 [56.4] (37.7-83.0) & $56.8[58.5](37.5-80.0)$ & 0.02 & 0.02 \\
\hline \multirow[t]{2}{*}{ Body Mass Index, $\mathrm{kg} / \mathrm{m}^{2}$} & B & $22.0[21.2](14.5-39.1)$ & $22.8[21.9](16.5-37.5)$ & $23.0[22.1](17.2-37.1)$ & 0.01 & $<0.01$ \\
\hline & C & 20.4 [20.7] (12.7-29.1) & $20.8[20.6](15.4-28.1)$ & 21.2 [20.7] (15.4-29.7) & 0.01 & 0.02 \\
\hline \multirow[t]{2}{*}{ Oral calorie intake, kCal } & B & 744.7 [800] (0-1500) & 1190.5 [1200] (0-2550) & 1204.2 [1280] (0-2550) & 5.44 & $<0.01$ \\
\hline & C & $462.4[500](0-1300)$ & 642.1 [600] (0-1700) & 735.3 [600] (0-1900) & 3.03 & $<0.01$ \\
\hline \multirow[t]{2}{*}{ Oral protein intake, $\mathrm{g} /$ day } & $\mathrm{B}$ & $24.8[25](0-50)$ & $40.6[40](0-110)$ & 40.8 [45] (0-90) & 0.19 & $<0.01$ \\
\hline & C & $17.6[17.5](0-50)$ & $24.6[25](0-75)$ & $28.8[30](0-60)$ & 0.12 & $<0.01$ \\
\hline \multirow[t]{2}{*}{ Karnofsky PS } & B & $67.7[70](50-90)$ & NA & 74.5 [80] (50-90) & NA & $<0.01$ \\
\hline & C & $67.1[70](50-80)$ & NA & 70.6 [70] (50-90) & NA & 0.1 \\
\hline \multirow[t]{2}{*}{ ECOG PS } & B & $1.3[1](0-2)$ & NA & $1.0[1](0-2)$ & NA & $<0.01$ \\
\hline & C & $1.4[1](1-2)$ & NA & $1.3[1](0-2)$ & NA & 0.82 \\
\hline \multirow[t]{2}{*}{ Albumin, $\mathrm{g} / \mathrm{dL}$} & B & $3.4[3.5](1.9-4.5)$ & NA & $3.7[3.7](2.8-4.4)$ & NA & 0.02 \\
\hline & C & $3.4[3.5](1.9-4.4)$ & NA & $3.4[3.3](2.2-4.6)$ & NA & 0.70 \\
\hline \multirow[t]{2}{*}{ C-reactive protein, mg/L } & B & 17.7 [10.5] (0.4-71) & NA & 17.3 [4.0] (0.1-140) & NA & 0.41 \\
\hline & C & 15.7 [12.2] (0.9-73.2) & NA & $10.1[7.2](0.1-56.5)$ & NA & 0.11 \\
\hline \multirow[t]{2}{*}{ mGPS } & B & $1[1](0-2)$ & NA & $0.6[0](0-2)$ & NA & 0.07 \\
\hline & C & $1[1](0-2)$ & NA & $0.6[0](0-2)$ & NA & 0.10 \\
\hline \multicolumn{7}{|l|}{ BIA Variables } \\
\hline \multirow[t]{2}{*}{ Resistance, Ohm } & B & $560.0[550.0](401-720)$ & $573.5[586.0](401-722)$ & 567.3 [602.0] (407-698) & 0.10 & 0.42 \\
\hline & C & 619.0 [625] (402-804) & 598.4 [594] (405-898) & 599.1 [610.0] (372-780) & -0.24 & 0.12 \\
\hline \multirow[t]{2}{*}{ Reactance, Ohm } & B & 45.4 [42.0] (19-118) & 46.2 [42.6] (20-69) & $44.8[42.0](23-72)$ & 0.00 & 0.88 \\
\hline & C & 58.0 [53.0] (23-120) & 55.5 [52.0] (25-99) & $57.1[51.5](22-116)$ & -0.01 & 0.69 \\
\hline \multirow[t]{2}{*}{ Phase Angle, $^{\circ}$} & B & $4.5[4.2](2.5-7.5)$ & $4.6[4.5](2.5-6.2)$ & $4.5[4.4](2.3-6.3)$ & 0.00 & 0.82 \\
\hline & C & $4.8[4.8](2.5-8.1)$ & $5.0[4.7](2.5-11.4)$ & $5.0[4.5](2.3-10.0)$ & 0.00 & 0.30 \\
\hline \multirow[t]{2}{*}{ Total Body Water, \% } & $\mathrm{B}$ & 59.5 [59.5] (39.3-76.6) & $57.2[58.1](41.3-72.8)$ & $56.9[56.5](42.0-72.0)$ & -0.03 & $<0.01$ \\
\hline & C & 59.4 [60.1] (40.3-85.8) & $59.8[60.0](43.7-76.6)$ & 58.9 [59.0] (41.7-76.4) & 0.00 & 0.74 \\
\hline \multirow[t]{2}{*}{ Extracellular Water, \%, } & $\mathrm{B}$ & $54.1[56.1](25.4-70.7)$ & $53.8[53.8](44.9-69.9)$ & $54.4[54.4](44.2-73.2)$ & 0.00 & 0.89 \\
\hline & C & 50.4 [49.1] (31.7-68.5) & $50.5[50.5](29.5-69.2)$ & $50.2[51.5](32.3-71.3)$ & 0.00 & 0.93 \\
\hline \multirow[t]{2}{*}{ Intracellular water, \% } & B & 45.9 [43.9] (29.3-74.6) & 46.6 [46.3] (30.1-55.1) & 45.6 [45.6] (26.8-55.8) & 0.00 & 0.95 \\
\hline & C & $49.6[51.0](31.5-68.3)$ & 49.5 [49.6] (30.8-70.5) & 49.5 [48.0] (28.7-67.7) & 0.00 & 0.89 \\
\hline \multirow[t]{2}{*}{ Fat Mass, \% } & B & $23.8[25.4](4.6-50.8)$ & $26.2[26.9](0.5-48.4)$ & 28.5 [29.1] (1.6-76.9) & 0.05 & 0.02 \\
\hline & C & $22.8[22.7](-7.3-45.0)$ & $22.1[20.5](1.2-45.4)$ & 23.4 [21.6] (3.3-43.0) & 0.00 & 0.78 \\
\hline \multirow[t]{2}{*}{ Fat Free Mass, \% } & B & $74.4[74.5](49.2-86.9)$ & 73.8 [73.1] (51.6-99.5) & 73.2 [72.0] (52.2-98.4) & -0.01 & 0.401 \\
\hline & C & 75.0 [77.4] (55.0-85.4) & 77.9 [79.6] (54.6-101.2) & 76.6 [78.5] (57.0-96.7) & 0.02 & 0.09 \\
\hline \multirow[t]{2}{*}{ Muscle Mass, \% } & B & $42.6[37.7](25.7-144.6)$ & $38.8[36.9](27.3-64.5)$ & $38.2[35.6](24.2-79.9)$ & -0.05 & 0.20 \\
\hline & C & 45.2 [44.8] (25.3-98.1) & $47.3[44.6](19.5-120.7)$ & 45.6 [42.0] (23.5-81.3) & 0.01 & 0.69 \\
\hline \multirow[t]{2}{*}{ ECM/BCM Index, \% } & B & $1.6[1.6](-0.4-3.5)$ & $1.6[1.5](0.4-3.5)$ & $1.7[1.5](0.3-4.0)$ & 0.00 & 0.82 \\
\hline & C & $1.3[1.2](0.3-3.8)$ & $1.3[1.3](-0.1-3.7)$ & $1.3[1.2](0.2-3.7)$ & 0.00 & 0.69 \\
\hline
\end{tabular}

All values are expressed as median [median] (range), or percentage as indicated

T0: at start of HPN; T1: 60 days after the start of HPN; T2: 90 days after the start of HPN

BIA Bioelectrical impedance analysis, PG-SGA Patient-generated subjective global assessment; Category $\mathrm{A}=$ well-nourished; Category $\mathrm{B}=$ suspected malnutrition or moderate malnutrition; Category $C$ = severely malnourished; NA Not applicable, PS Performance status, mGPS Modified Glasgow prognostic score 


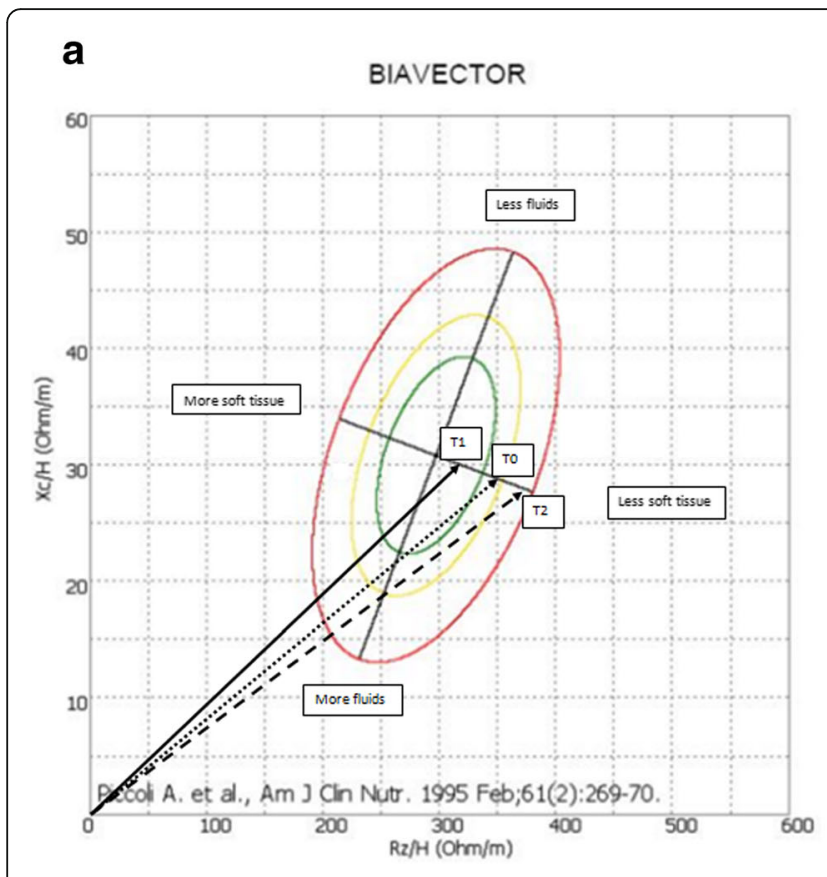

\section{b}

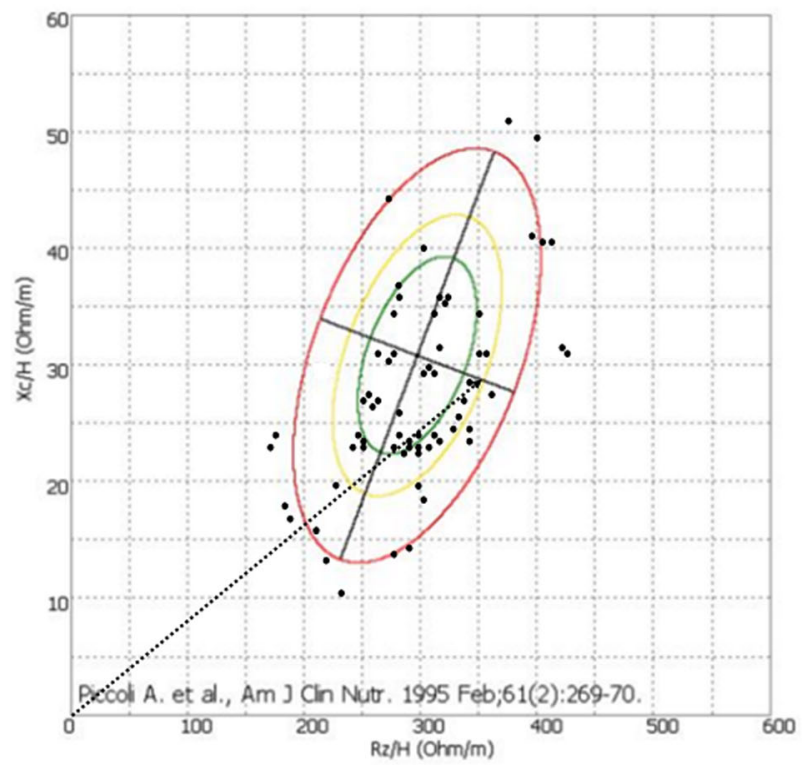

d

BIAVECTOR T2
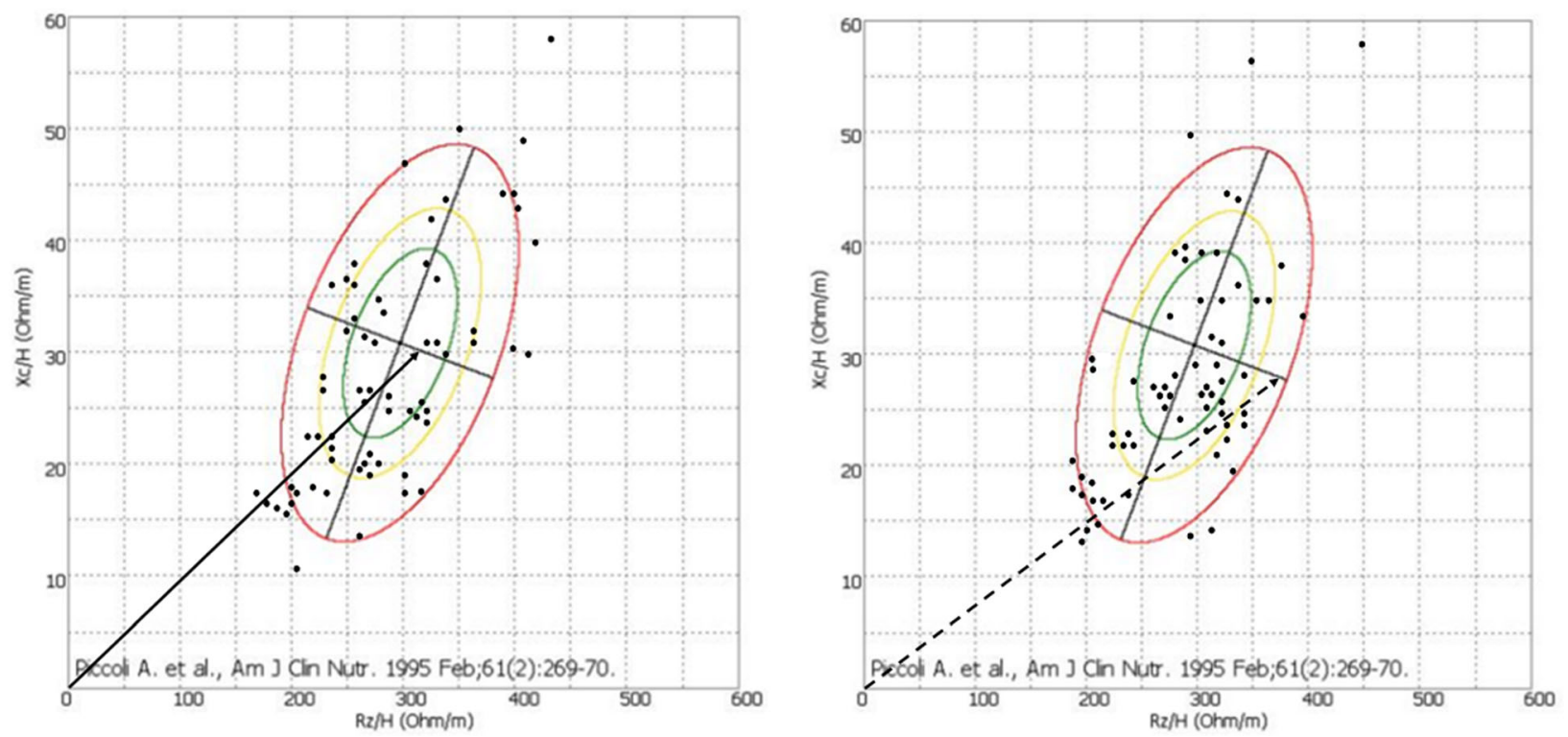

Fig. 1 a BIAVECTOR at TO, T1, and T2. BIA, bioelectrical impedance analysis; TO, baseline, T1 60 and T2 90 days after the start of home parenteral nutrition; XC, reactance; RZ, resistance, H, height. b BIAVECTOR at the start of HPN (TO). BIA, bioelectrical impedance analysis; HPN, home parenteral nutrition; XC, reactance; $R Z$, resistance, $H$, height. c BIAVECTOR 60 days after the start of HPN (T1). BIA, bioelectrical impedance analysis; HPN, home parenteral nutrition; $X C$, reactance; $R Z$, resistance, $H$, height. d BIAVECTOR 90 days after the start of HPN (T2). BIA, bioelectrical impedance analysis; HPN, home parenteral nutrition; $X C$, reactance; $R Z$, resistance, $H$, height

clinicians more accurately interpret changes in weight. Protein loss is a challenge to reverse, and early PN seems to stabilize protein levels [9]. In the current study, HPN not only slowed the weight loss expected in the patient population, but patients experienced significant increases in weight as well as in BCM and a decrease in TBW. Considering this decrease in TBW, the increase in weight patients experienced cannot be simply attributed to an increase in body water.

BIA has also shown utility as a prognostic tool in survival for cancer patients receiving chemotherapy [47]. In the current study, there was an association between survival and reactance at baseline, potentially indicating intact cell membranes; an association with resistance at 


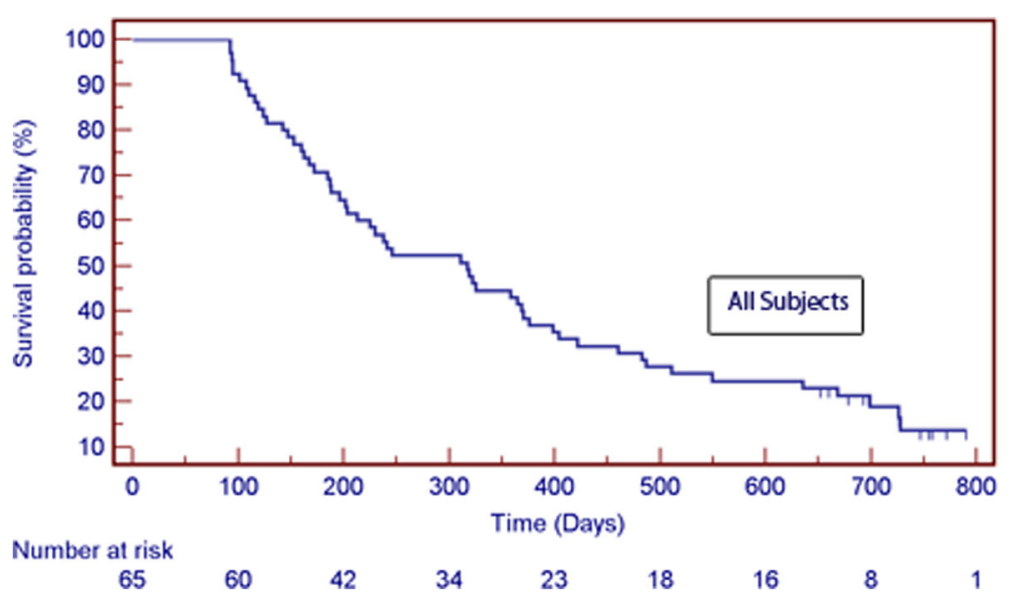

Fig. 2 Kaplan-Meier overall survival curve

60 days after the start of HPN; and with phase angle at 90 days after the start of HPN. BIA results vary based on age, gender and disease state [29], and although not all variables showed a correlation at all time points, longitudinal assessment of BIA can be a useful addition to provide better management of an oncology patient. Several previous studies have found no linear relationship between BIA variables and survival time [26, 32, 48], and one limitation of BIA noted is high interpatient variability [47]. Nevertheless, with interpretation in the context of an individual patient, BIA may offer a convenient, validated, non-invasive method of assessing nutritional status and survival [47].

Subjective assessment of nutritional status is commonly used to supplement objective measures, and PG-SGA has been shown to be effective in assessing nutritional status in cancer patients $[49,50]$. At the initiation of the study,

Table 3 Cox regression analysis of BIA variables associated with survival

\begin{tabular}{llll}
\hline Covariate & SE & $P$ & $95 \% \mathrm{Cl}$ \\
\hline T0 & & & \\
Reactance & 0.036 & $\mathbf{0 . 0 2}$ & $1.012-1.167$ \\
$\quad$ Resistance & 0.118 & 0.05 & $0.630-1.001$ \\
$\quad$ Phase angle & 0.555 & 0.10 & $0.135-1.189$ \\
T1 & & & \\
$\quad$ Reactance & 0.127 & 0.66 & $0.736-1.212$ \\
$\quad$ Resistance & 0.009 & $\mathbf{0 . 0 4}$ & $1.001-1.037$ \\
$\quad$ Phase angle & 1.134 & 0.44 & $0.260-22.161$ \\
T2 & & & \\
Reactance & 0.133 & 0.06 & $0.989-1.663$ \\
Resistance & 0.017 & 0.67 & $0.959-1.027$ \\
Phase angle & 1.470 & $\mathbf{0 . 0 5}$ & $1.130-1.591$ \\
\hline
\end{tabular}

T0: at start of HPN; T1: 60 days after the start of HPN; T2: 90 days after the start of HPN

BIA Bioelectrical impedance analysis, SE Standard error, CI Confidence interval all patients were rated as moderately to severely malnourished. After 90 days of HPN, over 30\% of patients experienced an improvement in PG-SGA assessment, with 5 patients reaching well-nourished status. The results of this study are consistent with previous research showing not only the utility of PG-SGA to assess nutritional risk, but also its value as a predictor of mortality in cancer patients [50]. In the current study, survival was correlated with PG-SGA rating at 90 days, with patients rated as poorly nourished having the lowest probability of survival. Patients who improved to a rating of well-nourished had the longest median survival in rank order, followed by patients who were consistently rated moderately malnourished at baseline and at 90 days. Shortest median survival times were seen in patients who declined from moderately malnourished to severely malnourished followed by patients who were consistently rated severely malnourished at baseline and at 90 days. These results highlight the impact of HPN in improving nutritional status, which can lead to improved survival. They also reinforce the importance of intervening with HPN in cancer patients before they reach severe malnourishment. Early intervention with HPN is reinforced by the study results, as patients who began HPN with PG-SGA rating of B experienced more significant improvements in clinical and BIA parameters than patients who began HPN with PG-SGA rating of C.

HPN can improve patient performance status, as demonstrated by the increase in KPS after 90 days of HPN seen in this study. There was a median increase in KPS of 10 after 90 days of HPN, and the proportion of patients with KPS $>70$ increased by $16 \%$. Functional status is an important factor in oncology patient QoL; moving from a KPS of 70 to 80 means the difference in being able to carry out normal activities with no special care needed. In addition to the impact on daily living, a higher KPS has been associated with better survival in cancer patients $[16,24]$. 

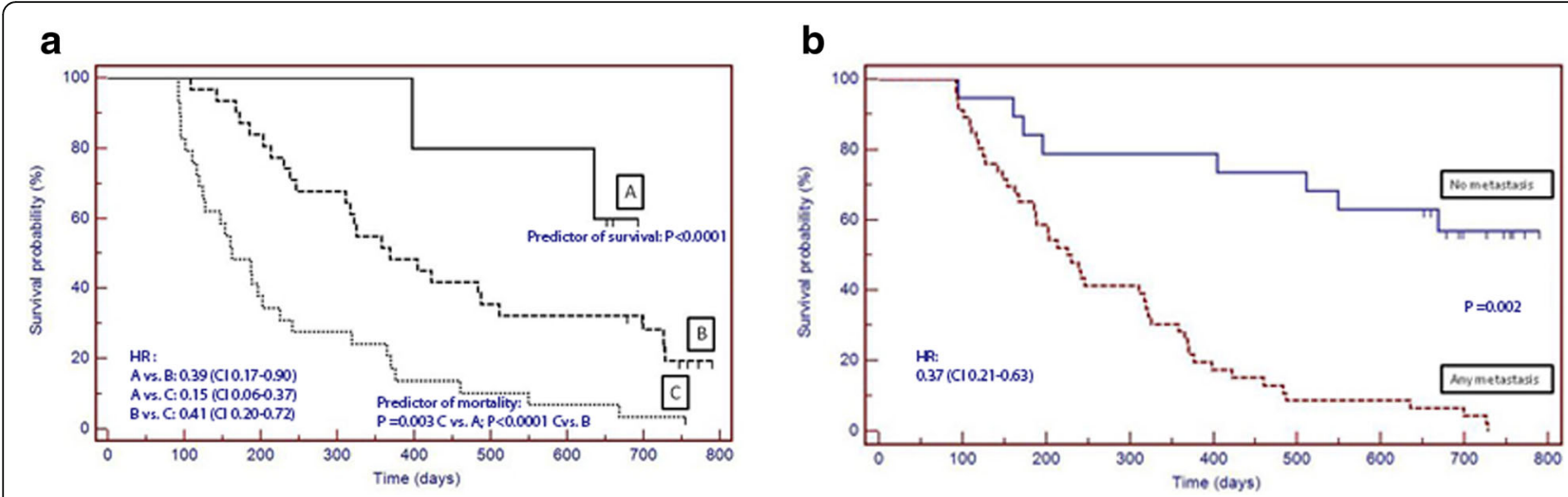

Fig. 3 a Log rank test Survival curve for PG-SGA at T2. PG-SGA category A at T2 was a predictor of survival (median duration days: 652; Cl 635-660; $P<0.0001$ ); PG-SGA category C (median duration days: 162; Cl 102-222) was a predictor of mortality vs. both $A$ and $B$ categories $(P=0.003$ and $P<0.0001$, respectively). The HR for PG-SGA category as a predictor of survival for category A vs. B was 0.39 (Cl 0.17-0.90); for category A vs. C was 0.15 (Cl 0.06-0.37), and for category B vs. C was 0.41 (Cl 0.20-0.72). PG-SGA, Patient-Generated Subjective Global Assessment; Category A = wellnourished; Category $B=$ suspected malnutrition or moderate malnutrition; Category $C=$ severely malnourished; $\mathrm{T} 2,90$ days after the start of home parenteral nutrition. b Log-rank test Survival curve for Metastasis. No metastasis (median duration days: 487; Cl 88-894) vs. any metastasis (median duration days: 213; $\mathrm{Cl} 163-263)$ was a predictor of survival $(P=0.002)$. The $\mathrm{HR}$ for no metastasis as a predictor of survival was $0.37(\mathrm{Cl} 0.21-0.63)$

Evidence shows systemic inflammatory response is associated with weight loss, loss of muscle tissue, and decreased functional status, and that inflammation scales can identify risk in cancer patients. [24, 38] The prognostic value of mGPS in cancer patients has been shown in multiple studies [51, 52]. Proctor et al. noted an association between an mGPS of 2, (i.e., the worst value from a prognostic point of view) and a $160 \%$ reduction in survival, regardless of tumor site [52]. In the current study, there was a $32 \%$ reduction in the proportion of patients categorized as mGPS of 2 after 90 days of HPN.

Over $70 \%$ of patients enrolled in the study had metastases. Research previously conducted showed that survival of cancer patients on HPN was affected by tumor spread [24], and the current study supports this finding, with metastasis as a predictor of survival.

Table 4 Survival by change in PG-SGA from T0 to T2

\begin{tabular}{|c|c|c|}
\hline & N (\%) & $\begin{array}{l}\text { Survival } \\
\text { Median (range) }\end{array}$ \\
\hline \multicolumn{3}{|l|}{ Improved } \\
\hline$B$ to $A$ & $5(7.7 \%)$ & $652(398-693)$ \\
\hline C to $B$ & $15(23.1 \%)$ & $311(108-772)$ \\
\hline \multicolumn{3}{|c|}{ Stayed the Same } \\
\hline$B$ to $B$ & $16(24.6 \%)$ & $452(230-790)$ \\
\hline$C$ to $C$ & 19 (29.2\%) & $162(92-765)$ \\
\hline \multicolumn{3}{|c|}{ Declined } \\
\hline B to $C$ & 10 (15.4\%) & $156(92-376)$ \\
\hline
\end{tabular}

\section{Strengths and limitations of the study}

The strength of this study is that it prospectively enrolled a population of adult patients with many similar characteristics who all: (1) had solid tumors of advanced stages; (2) were malnourished; (3) were outpatients; (4) were receiving chemotherapy during the course of the study; (5) were managed with daily HPN and received the same protocol of care at home. Moreover, no patients were lost to follow-up, and all patients survived through the day 90 assessment.

This study has several limitations. The most important is the lack of a comparator group to assess the effects of HPN; however, considering guidelines recommending supplemental nutrition in malnourished cancer patients receiving chemotherapy [20] it would have been ethically unacceptable to have a non-HPN control arm. The second limitation is the relatively small number of enrolled patients. However, with respect to the power of statistical analyses, a sample of 52 patients would allow detection of correlations between BIA measures and survival with $80 \%$ power and $\alpha=0.05$. Because the distribution of data for BIA measures is often skewed, to reduce the type I error rate a greater number of patients was enrolled. Also, the series of patients are heterogeneous and diverse cancers are rather different in terms of nutritional aspects. However, we investigated the nutritional status of this population in-depth with two well-accepted and effective scores (i.e., PG-SGA and mGPs). Third, our intention was not to assess QoL because it was already evaluated in a previous study [43]. Finally, a detailed description of the chemotherapy and radiotherapy regimens adopted in the different sites and stages of the tumors as well as the associated toxicity was beyond the aims of this paper. 


\section{Conclusions}

To the best of our knowledge, this is the first longitudinal study investigating the impact of HPN in cancer patients receiving chemotherapy by an evaluation of body composition using BIA measures. Our study showed that malnourished patients with advanced cancer experienced significantly improved body weight, BMI, oral calorie and protein intake. After 90 days of HPN, patients had significantly improved nutritional status, some BIA measures, performance status and prognostic score. Moreover, the BIA measures reactance, resistance and phase angle were significantly associated with survival. Finally, survival was correlated with PG-SGA rating at 90 days, with patients rated as poorly nourished having the lowest probability of survival.

HPN may be an important therapy in oncology patients receiving chemotherapy. Nowadays, clinicians must use tools to identify patients early who are likely to benefit from HPN, and assist in ongoing management. Longitudinal use of BIA may help track the impact of HPN and disease progression, potentially contributing to a better management of global patient care.

\section{Abbreviations \\ BCM: Body cell mass; BIA: Bioelectrical impedance analysis; BIVA: Bioelectrical impedance vector analysis; BMl: Body mass index; ECM: Extracellular mass; ECOG: Eastern Cooperative Oncology Group; ECW: Extracellular water; FFM: Fat free mass; FM: Fat mass; GPS: Glasgow prognostic score; HPN: Home parenteral nutrition; IW: Intracellular water; KPS: Karnofsky performance status; mGPS: Modified Glasgow Prognostic Score; PA: Phase angle; PG-SGA: Patient-Generated Subjective Global Assessment; PN: Parenteral nutrition; QoL: Quality of life; R: Resistance; ROC: Receiver Operating Characteristic; TBW: Total body water; XC: Reactance; Z: Impedance}

\section{Acknowledgements}

The authors wish to thank Maria Paluselli for providing copyediting and editorial assistance.

\section{Funding}

The authors acknowledge copyediting and editorial assistance provided by Maria Paluselli, and supplementary statistical support provided by DZS Clinical Services, which were funded by Baxter Healthcare Corporation and directed solely by the manuscript authors. Baxter Healthcare Corporation did not participate in the design of the study, data collection, analysis, interpretation, or writing of the manuscript.

\section{Availability of data and materials}

The dataset of this study is deposited in the repositories of Regional Public Healthcare Office (Regione Piemonte) according to our Institutional Review Board/Human Subjects Research Committee (Comitato Etico Interaziendale A.O.U. San Giovanni Battista di Torino - A.O. C.T.O. Maria Adelaide di Torino) requirements for research projects and is available on request.

\section{Authors' contributions}

PC performed study design, collected the data, performed data analysis, and wrote the manuscript. TM performed study design, collected the data, collaborated to data analysis and manuscript writing. MF performed data analysis. ADF revised the manuscript critically for important intellectual content. All authors read and approved the final manuscript.

\section{Ethics approval and consent to participate}

The study protocol was reviewed and approved by our Institutional Ethics Committee (Comitato Etico Interaziendale A.O.U. San Giovanni Battista di
Torino - A.O. C.T.O. Maria Adelaide di Torino) and patients provided written informed consent before their enrollment. The consent to participate was obtained from PC. The work was conducted in compliance with Institutional Review Board/Human Subjects Research Committee requirements.

\section{Consent for publication}

Not applicable.

\section{Competing interests}

P. Cotogni reports honoraria for speaking and teaching from Baxter. The other authors declare that they have no competing interests.

\section{Publisher's Note}

Springer Nature remains neutral with regard to jurisdictional claims in published maps and institutional affiliations.

\section{Author details}

${ }^{1}$ Department of Anaesthesia and Intensive Care, Pain Management and Palliative Care, S. Giovanni Battista Hospital, University of Turin, C.so Bramante 88, 10126 Turin, Italy. ${ }^{2}$ Unit of Parenteral Nutrition in Oncology, S. Giovanni Battista Hospital, C.so Bramante 88, 10126 Turin, Italy. ${ }^{3}$ Clinical Nutrition, S. Giovanni Battista Hospital, C.so Bramante 88, 10126 Turin, Italy.

Received: 22 December 2017 Accepted: 4 October 2018

Published online: 17 October 2018

\section{References}

1. Hébuterne $X$, Lemarié $E$, Michallet, et al. Prevalence of malnutrition and current use of nutrition support in patients with cancer. JPEN. 2014;38: 196-204.

2. Lis CG, Gupta D, Lammersfeld CA, Markman M, Vashi PG. Role of nutritional status in predicting quality of life outcomes in cancer-a systematic review of the epidemiological literature. Nutr J. 2012;11:27.

3. Fearon K, Strasser F, Anker SD, et al. Definition and classification of cancer cachexia: an international consensus. Lancet Oncol. 2011:12:489-95.

4. Rier HN, Jager A, Sleijfer S, Maier AB, Levin MD. The prevalence and prognostic value of low muscle mass in Cancer patients: a review of the literature. Oncologist. 2017;22:901-9.

5. Prado CM, Baracos VE, McCargar LJ, et al. Sarcopenia as a determinant of chemotherapy toxicity and time to tumor progression in metastatic breast cancer patients receiving capecitabine treatment. Clin Cancer Res. 2009;15: 2920-6.

6. Prado CM, Antoun S, Sawyer MB, Baracos VE. Two faces of drug therapy in cancer: drug-related lean tissue loss and its adverse consequences to survival and toxicity. Curr Opin Clin Nutr Metab Care. 2011;14:250-4.

7. Bozzetti F. Nutritional support of the oncology patient. Crit Rev Oncol Hematol. 2013;87:172-200

8. Attar A, Malka D, Sabate JM, et al. Malnutrition is high and underestimated during chemotherapy in gastrointestinal cancer: an AGEO prospective crosssectional multicenter study. Nutr Cancer. 2012;64:535-42.

9. Prado CM, Sawyer MB, Ghosh S, et al. Central tenet of cancer cachexia therapy: do patients with advanced cancer have exploitable anabolic potential? Am J Clin Nutr. 2013;98:1012-9.

10. Caccialanza R, De Lorenzo F, Gianotti L, et al. Nutritional support for cancer patients: still a neglected right? Support Care Cancer. 2017;25:3001-4.

11. Soo I, Gramlich L. Use of parenteral nutrition in patients with advanced cancer. Appl Physiol Nutr Metab. 2008:33:102-6.

12. Brard L, Weitzen $S$, Strubel-Lagan SL, et al. The effect of total parenteral nutrition on the survival of terminally ill ovarian cancer patients. Gynecol Oncol. 2006;103:176-80.

13. Fan BG. Parenteral nutrition prolongs the survival of patients associated with malignant gastrointestinal obstruction. JPEN J Parenter Enteral Nutr. 2007:31:508-10.

14. Wang MY, Wu MH, Hsieh DY, et al. Home parenteral nutrition support in adults: experience of a medical center in Asia. JPEN J Parenter Enteral Nutr. 2007;31:306-10.

15. Pelzer U, Arnold D, Gövercin M, et al. Parenteral nutrition support for patients with pancreatic cancer. Results of a phase II study. BMC Cancer. 2010;10:86. 
16. Chermesh I, Mashiach T, Amit A, et al. Home parenteral nutrition (HTPN) for incurable patients with cancer with gastrointestinal obstruction: do the benefits outweigh the risks? Med Oncol. 2011;28:83-8.

17. Richter E, Denecke A, Klapdor S, Klapdor R. Parenteral nutrition support for patients with pancreatic cancer-improvement of the nutritional status and the therapeutic outcome. Anticancer Res. 2012;32:2111-8.

18. Bozzetti F, Santarpia L, Pironi L, et al. The prognosis of incurable cachectic cancer patients on home parenteral nutrition: a multi-Centre observational study with prospective follow-up of 414 patients. Ann Oncol. 2014;25:487-93.

19. Staun $M$, Pironi $L$, Bozzetti $F$, et al. ESPEN guidelines on parenteral nutrition: home parenteral nutrition (HPN) in adult patients. Clin Nutr. 2009;28:467-79.

20. Arends J, Bachmann P, Baracos V, et al. ESPEN guidelines on nutrition in cancer patients. Clin Nutr. 2017;36:11-48.

21. August DA, Huhmann MB, American Society for Parenteral and Enteral Nutrition (A.S.P.E.N.) Board of Directors. A.S.P.EN clinical guidelines: nutrition support therapy during adult anticancer treatment and in hematopoietic cell transplantation. JPEN J Parenter Enteral Nutr. 2009;33:472-500.

22. Cotogni P. Enteral versus parenteral nutrition in cancer patients: evidences and controversies. Ann Palliat Med. 2016;1:42-9.

23. Martin L, Senesse P, Gioulbasanis I, et al. Diagnostic criteria for the classification of cancer-associated weight loss. J Clin Oncol. 2015;33:90-9.

24. Bozzetti F, Cotogni P, Lo Vullo S, Pironi L, Giardiello D, Mariani L. Development and validation of a nomogram to predict survival in incurable cachectic cancer patients on home parenteral nutrition. Ann Oncol. 2015;26: 2335-40.

25. Choi Y, Oh DY, Kim TY, et al. Skeletal muscle depletion predicts the prognosis of patients with advanced pancreatic Cancer undergoing palliative chemotherapy, independent of body mass index. PLoS One. 2015; 10:e0139749.

26. Crawford GB, Robinson JA, Hunt RW, Piller NB, Esterman A. Estimating survival in patients with Cancer receiving palliative care: is analysis of body composition using bioimpedance helpful? J Palliat Med. 2009;12:1009-14.

27. Grossberg AJ, Chamchod S, Fuller CD, et al. Association of Body Composition with Survival and Locoregional Control of radiotherapy-treated head and neck squamous cell carcinoma. JAMA Oncol. 2016:2:782-9.

28. Barbosa-Silva MC, Barros AJ. Bioelectrical impedance analysis in clinical practice: a new perspective on its use beyond body composition equations. Curr Opin Clin Nutr Metab Care. 2005;8:311e7.

29. Bosy-Westphal A, Danielzik S, Dörhöfer RP, Piccoli A, Müller MJ. Patterns of bioelectrical impedance vector distribution by body mass index and age: implications for body-composition analysis. Am J Clin Nutr. 2005;82:60-8.

30. Nwosu AC, Morris L, Mayland C, Mason S, Pettitt A, Ellershaw J. Longitudinal bioimpedance assessments to evaluate hydration in POEMS syndrome. BMJ Support Palliat Care. 2016;6:369-72.

31. Halpern-Silveira D, Susin LRO, Borges LR, Paiva SI, Assunção MCF, Gonzalez MD. Body weight and fat-free mass changes in a cohort of patients receiving chemotherapy. Support Care Cancer. 2010;18:617-25.

32. Gupta D, Lis CG, Dahlk SL, et al. The relationship between bioelectrical impedance phase angle and subjective global assessment in advanced colorectal cancer. Nutr J. 2008;7:19-25.

33. Norman K, Stobäus N, Zocher D, et al. Cutoff percentiles of bioelectrical phase angle predict functionality, quality of life, and mortality in patients with cancer. Am J Clin Nutr. 2010;92:612-9.

34. Norman K, Stobäus N, Pirlich M, Bosy-Westphal A. Bioelectrical phase angle and impedance vector analysis: clinical relevance and applicability of impedance parameters. Clin Nutr. 2012;31:854-61.

35. Cotogni P, Pittiruti M, Barbero C, et al. Catheter-related complications in cancer patients on home parenteral nutrition: a prospective study of over 51,000 catheter days. JPEN J Parenter Enteral Nutr. 2013;37:375-83.

36. Oken MM, Creech RH, Tormey DC, et al. Toxicity and response criteria of the eastern cooperative oncology group. Am J Clin Oncol. 1982;5:649-55.

37. Karnofsky DA, Burchenal JH. The clinical evaluation of chemotherapeutic agents in cancer. In: MacLeod CM, editor. Evaluation of chemotherapeutic agents. New York: Columbia University Press; 1949. p. 191-205.

38. McMillan DC. An inflammation-based prognostic score and its role in the nutrition-based management of patients with cancer. Proc Nutr Soc. 2008; 67:257-62.

39. Bauer J, Capra S, Ferguson M. Use of the scored patient-generated subjective global assessment (PG-SGA) as a nutrition assessment tool in patients with cancer. Eur J Clin Nutr. 2002;56:779-85.
40. Kyle UG, Bosaeusb I, De Lorenzo AD, et al. ESPEN working group. Bioelectrical impedance analysis part I: review of principles and methods. Clin Nutr. 2004;23:1226-43.

41. Bioelectrical impedance analysis in body composition measurement: National Institutes of Health technology assessment conference statement. Am J Clin Nutr. 1996:64:524S-32S.

42. Vashi PG, Dahlk S, Popiel B, Lammersfeld CA, Ireton-Jones C, Gupta D. A longitudinal study investigating quality of life and nutritional outcomes in advanced cancer patients receiving home parenteral nutrition. BMC Cancer. 2014;14:593

43. Cotogni $P$, De Carli L, Passera $R$, et al. Longitudinal study of quality of life in advanced cancer patients on home parenteral nutrition. Cancer Med. 2017; 7:1799-806.

44. Bozzetti F, Cozzaglio L, Biganzoli E, et al. Quality of life and length of survival in advanced cancer patients on home parenteral nutrition. Clin Nutr. 2002;21:281-8

45. Culine S, Chambrier C, Tadmouri A, et al. Home parenteral nutrition improves quality of life and nutritional status in patients with cancer: a French observational multicentre study. Support Care Cancer. 2014;22:1867-74.

46. Antoun S, Borget I, Lanoy E. Impact of sarcopenia on the prognosis and treatment toxicities in patients diagnosed with cancer. Curr Opin Support Palliat Care. 2013:7:383-9.

47. Grundmann O, Yoon SL, Williams JJ. The value of bioelectrical impedance analysis and phase angle in the evaluation of malnutrition and quality of life in cancer patients--a comprehensive review. Eur J Clin Nutr. 2015;69:1290-7.

48. Gupta D, Lammersfeld CA, Vashi PG, et al. Bioelectrical impedance phase angle as a prognostic indicator in breast cancer. BMC Cancer. 2008;8:24955.

49. Li R, Wu J, Ma M, et al. Comparison of PG-SGA, SGA and body-composition measurement in detecting malnutrition among newly diagnosed lung cancer patients in stage IIIB/IV and benign conditions. Med Oncol. 2011;28: 689-96.

50. Rodrigues CS, Lacerda MS, Chaves GV. Patient generated subjective global assessment as a prognosis tool in women with gynecological cancer. Nutrition. 2015:31:1372-8.

51. Dolan RD, MCSorley ST, Horgan PG, Laird B, McMillan DC. The role of the systemic inflammatory response in predicting outcomes in patients with advanced inoperable cancer: systematic review and meta-analysis. Crit Rev Oncol Hematol. 2017;116:134-46.

52. Proctor MJ, Morrison DS, Talwar K, et al. An inflammation-based prognostic score (mGPS) predicts cancer survival independent of tumour site: a Glasgow inflammation outcome study. Br J Cancer. 2011;104:726-34.

Ready to submit your research? Choose BMC and benefit from

- fast, convenient online submission

- thorough peer review by experienced researchers in your field

- rapid publication on acceptance

- support for research data, including large and complex data types

- gold Open Access which fosters wider collaboration and increased citations

- maximum visibility for your research: over $100 \mathrm{M}$ website views per year

At $\mathrm{BMC}$, research is always in progress.

Learn more biomedcentral.com/submissions 